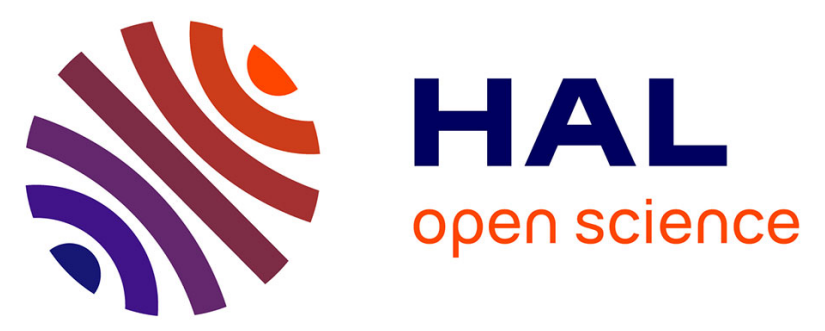

\title{
Diego, Asie et Amérique : comment la répartition d'un seul groupe sanguin suffit à décrire le peuplement de deux continents
}

\author{
Stéphane Mazières, Caroline Costedoat
}

\section{- To cite this version:}

Stéphane Mazières, Caroline Costedoat. Diego, Asie et Amérique : comment la répartition d'un seul groupe sanguin suffit à décrire le peuplement de deux continents. C.N.R.S. Editions. Le sport-santé / Os, sangs, gènes et cultures, $N^{\circ} 17$ (1), pp.133, 2019, CORPS, 9782271134837. 10.3917/corp1.017.0133 . hal-02996020

\section{HAL Id: hal-02996020 \\ https://hal.science/hal-02996020}

Submitted on 9 Nov 2020

HAL is a multi-disciplinary open access archive for the deposit and dissemination of scientific research documents, whether they are published or not. The documents may come from teaching and research institutions in France or abroad, or from public or private research centers.
L'archive ouverte pluridisciplinaire HAL, est destinée au dépôt et à la diffusion de documents scientifiques de niveau recherche, publiés ou non, émanant des établissements d'enseignement et de recherche français ou étrangers, des laboratoires publics ou privés. 


\section{Diego, Asie et Amérique : comment la répartition d'un seul groupe sanguin suffit à décrire le peuplement de deux continents}

Stéphane MAZIÈRES

Caroline COSTEDOAT

Le sang est depuis longtemps un objet anthropologique qui fédère les sciences biologiques, humaines et sociales (Bernard, 1983). La biologie du sang ne cesse de décrire de plus en plus finement les différents constituants qui le composent. Depuis 1901 et la découverte des groupes sanguins, ce sont à ce jour 36 systèmes, dont un quart a été décrit durant la seule décennie des années 1960, qui permettent de définir les différents " groupes sanguins ». Certains sangs sont compatibles, d'autres ne le sont pas. Dans la pensée de tout un chacun, les groupes sanguins désignent implicitement ceux du globule rouge. Les plus médiatiques sont évidemment les plus étudiés de par leur implication médicale : $\mathrm{ABO}$ et Rhésus. Toutefois, la stricte définition de ces marqueurs biologiques du sang et du soi désigne aussi les systèmes de groupes sanguins portés par des cellules autres que le globule rouge : globules blancs, plaquettes, etc. Pour la suite du présent essai, nous traiterons des systèmes de groupes sanguins du globule rouge, dits érythrocytaires, mais utiliserons la désignation simplifiée de groupes sanguins tout en gardant à l'esprit qu'il existe toutefois d'autres groupes sanguins ailleurs que sur le globule rouge.

L'objet des lignes qui vont suivre va être de montrer, à travers l'exemple d'un seul système de groupe sanguin, « Diego ", combien des marqueurs biologiques quelque peu délaissés par la communauté des anthropologues depuis l'avènement de la biologie moléculaire, sont toujours aussi pertinents pour remonter la généalogie des patrimoines génétiques des populations et pour raconter l'histoire des peuplements. 


\section{Pourquoi les groupes sanguins sont des marqueurs de choix pour les anthropologues biologistes}

Les groupes sanguins rassemblent l'ensemble de polymorphismes immunogènes du globule rouge, du globule blanc, des plaquettes, des neutrophiles et des immunoglobulines. Certaines de ces molécules tapissent également la couche des vaisseaux sanguins en contact avec le sang, les tissus des organes et peuvent être sécrétées dans les fluides comme la salive. Leur propriété de réaction antigène-anticorps leur confère une évidente implication médicale en transfusion sanguine, greffe et grossesse (Bailly et al., 2015). Pour l'Anthropologie biologique et génétique, qui doit beaucoup à la Médecine transfusionnelle, l'Hématologie, et la Parasitologie, la découverte et l'étude des systèmes de groupes sanguins menaient aux constats suivants : l'unicité biologique de chaque personne ${ }^{1}$, l'inanité du concept de race et l'inégale répartition des groupes sanguins entre les populations. Les systèmes de groupes sanguins ont activement participé aux débats anthropologiques sur la diversité biologique des populations humaines depuis le Out of Africa. Les principales problématiques auxquelles ils semblent apporter des réponses concernent la phylogénie, la primatologie, l'origine africaine de l'Homme anatomiquement moderne, la génétique en tant que science de l'hérédité, la génétique des populations, c'est-à-dire l'étude de l'évolution des pourcentages des caractères biologiques sur plusieurs générations, les relations hommes/pathogènes, l'étude de la coévolution gène/culture et enfin, l'objet de notre point de vue, l'étude des peuplements anciens.

Depuis cinq ans, les systèmes de groupes sanguins connaissent un regain d'intérêt pour deux raisons principales : l'évolution de l'Anthropologie biologique et la non-résolution de questions cruciales. Le bilan bibliométrique depuis 1960 montre que les groupes sanguins, comme l'ensemble des polymorphismes des protéines et des enzymes du sang, sont sortis des débats anthropologiques depuis l'avènement de méthodes en laboratoire offrant une appréciation plus résolutive de la variabilité biologique humaine actuelle et ancienne (marqueurs ADN, CT-scan, morphologie géométrique) et de son traitement informatique. Ceci est paradoxal car dans le même temps, il demeure de nombreuses questions quant à leur répartition, leur rôle biologique précis et leur histoire évolutive. Or justement, les quelques incursions scientifiques dans la répartition des groupes sanguins à la lumière des méthodes actuelles, sur des échantillons actuels et anciens, parviennent à résoudre des questions soulevées il y a 50 ans, comme l'origine de la prévalence du groupe $\mathrm{O}+$ en Amérique Centrale et Sud, mais demeurant inexpliquées sans cet ajout moderne d'informations ${ }^{2}$. 
Par ailleurs, connaître la répartition des groupes sanguins présente un intérêt médical crucial notamment en ce qui concerne les transfusions sanguines dans le contexte actuel des migrations. En effet, nous sommes aujourd'hui face à deux problèmes majeurs en transfusions : l'incompatibilité biologique entre le donneur et le receveur, et la non-participation de l'ensemble de la population au don du sang. Dans le contexte actuel de mondialisation et de flux migratoires intenses, il est crucial de maintenir des stocks de produits sanguins en adéquation avec la diversité sanguine de la population. Il s'agit d'assurer l'autosuffisance quantitative et qualitative, et de remédier aux différences sanguines entre la population des donneurs et celle des receveurs. En France métropolitaine et d'Outre-Mer, cet écart sanguin concerne particulièrement les communautés d'origine subsaharienne (Afrique, Antilles, etc.), qui présentent un taux plus élevé d'atteintes du globule rouge comme la drépanocytose nécessitant donc plus de produits sanguins de remplacement. Or pour ces communautés, le don de sang n'est pas une priorité ou n'est pas forcément usuel dans la culture d'origine, voire fait l'objet de réticences. Or, la diversité des groupes sanguins n'est pas entièrement représentée par les poches de sang issues de la seule population de France métropolitaine. L'Anthropologie, par son approche multidisciplinaire, intervient alors à deux niveaux : culturel pour identifier ces «freins » au don du sang (Chiaroni et al., 2016), et biologique, pour décrire et comprendre la répartition des groupes sanguins qui posent problème. Et c'est là que réside tout l'intérêt d'une action conjointe entre la sérologie, la biologie moléculaire, la génétique des populations, l'histoire et les sciences humaines et sociales. Ainsi, l'étude de la répartition et de la diversité des groupes sanguins à travers le monde est un enjeu de santé publique. Appréhender la dynamique évolutive de cette répartition depuis la mise en place des peuplements jusqu'aux effets de sélection est de ce fait primordial.

\section{Diego finalise l'étude du peuplement de l'Amérique}

La biologie, d'abord sanguine puis moléculaire, contribuera au débat des modalités du peuplement initial de l'Amérique. C'est précisément un groupe sanguin, Diego, qui consolidera l'hypothèse de l'origine asiatique à l'ensemble des populations amérindiennes actuelles. L'histoire de l'étude du peuplement initial de l'Amérique débute dès les premières expéditions qui suivirent la découverte du continent par les Européens. Le nom " Amérique » apparaît dès 1507 sur l'Universalis Cosmographia de Martin Waldseemüller et l'origine de ses occupants et la voie de passage sont pressenties dès 1589 par le Jésuite José de 
Acosta : "Parce qu'il ne s'agit pas de ce que Dieu peut faire, mais de ce qui est conforme avec la raison, l'ordre et le style de l'humain [...] le simple monde qu'ils appellent les Indes n'est pas loin de l'autre, le bien connu [...] ils restent voisins [...] ainsi les Indiens sont passés de l'un à l'autre plus par voie de terre que par la mer [...]", puis en 1614 par Edward Brerewood, antiquaire, mathématicien, linguiste et humaniste : "Les peuples d'Amérique, n'ayant pas les arts civilisés de la Chine, l'Inde et le Japon, de toutes les nations de la Terre, ressemblent aux vieux et rudes Tartars ».

Le système des groupes sanguins de Diego a été le premier marqueur génétique, et peut-être le plus convaincant reliant les Amérindiens à la Sibérie. Ce système a été découvert en 1953 chez une femme vénézuélienne qui a subi trois anémies hémolytiques sévères du nouveau-né. Ces accidents obstétricaux étaient dus à un anticorps irrégulier, différent des neuf systèmes de groupes sanguins alors connus. Cet anticorps, ainsi que l'antigène ciblé, devaient alors constituer un nouveau système, le dixième. Il fut désigné " Diego » comme le nom marital de cette patiente. Il se compose essentiellement de deux formes de son antigène ; Diego a $\left(\mathrm{Di}^{\mathrm{a}}\right)$ et Diego b $\left(\mathrm{Di}^{\mathrm{b}}\right)$. La recherche de Diego dans les populations mondiales montrera que $\mathrm{Di}^{\mathrm{b}}$ est ubiquiste alors que $\mathrm{Di}^{\mathrm{a}}$ est uniquement présent chez les populations d'ascendance mongole, et notamment chez les groupes amérindiens d'Amérique du sud où $\mathrm{Di}^{\mathrm{a}}$ atteint les plus hautes fréquences dans le bassin amazonien (Junqueira et Castilho, 2002).
Nous sommes en 1957 et l'étude du peuplement connaîtra par la suite deux regains d'intérêt en Anthropologie Biologique. Tout d'abord dans les années 1980 avec la description de la diversité génétique sanguine des populations amérindiennes. En 1986, une étude mêlant protéines sanguines, variabilité dentaire et linguistique indiquait que le peuplement s'était réalisé en trois vagues (Greenberg et al. 1986). Ce modèle par son approche exhaustive, demeura le modèle incontournable et l'hypothèse de départ de l'ensemble des travaux qui suivirent. La deuxième phase d'intérêt intervient au tournant des années 1990 avec l'utilisation conjointe des marqueurs ADN et de la morphologie du crâne ${ }^{3}$. Si l'origine asiatique et la voie de passage par Béring ne sont plus discutées, c'est le nombre de migrations qui faisait - et fait encore grand débat (Mazières, 2011). Le modèle à trois vagues était alors contredit, avec une seule vague pour expliquer la diversité de l'ADN amérindien et la variabilité crânienne. Ce modèle d'un peuplement unique perdurera pendant deux décennies et l'utilisation d'une nouvelle technologie dans les années 2010, les puces à ADN qui permettent d'analyser en une seule fois plusieurs centaines de milliers de marqueurs de l'ADN, permirent d'appréhender cette problématique sous un nouveau regard. En 2012, une étude menée sur 52 populations prend le contre-pied et ravive le modèle tripartite de 1986 (Reich et al., 2012). Désormais, l'ensemble des travaux menés sur des échantillons amérindiens 
actuels et anciens du nord de l'Amérique $\mathrm{du}$ Nord convergent vers un peuplement en trois phases, étalées entre le dernier maximum glaciaire (15 000 ans) et le premier millénaire de notre ère. Dans cette actualité de recherche, dominée par la technologie moderne des puces et un peuplement du continent américain en 3 étapes, une étude rétrospective menée en 2015 dénote (Bégat et al., 2015). Elle repose non pas sur les nombreux marqueurs des puces ADN mais sur un seul, le groupe sanguin Diego et parvient aux mêmes conclusions.

L'antigène $\mathrm{Di}^{\mathrm{a}}$ atteint les plus hautes fréquences 40 à $50 \%$ dans le bassin amazonien. Il est par contre absent des populations subarctiques, Fuégiennes, Panaméennes, du Chaco et Yanomama du Brésil et du Venezuela. Une analyse plus fine montre que cette distribution en foyers ne relève pas du hasard. Les plus fortes fréquences sont précisément rencontrées chez les populations des rameaux linguistiques Equatorien-Tucanoén et Ge-Pano-Karib, alors que les plus faibles fréquences caractérisent les populations de langues Uto-Aztèque et Chibchan-Paezan. Lorsqu'on se penche sur les pratiques alimentaires possibles à l'époque précolombienne, les analyses montrent que l'antigène $\mathrm{Di}^{\mathrm{a}}$ est observé chez les populations anciennement de tradition chasseurs-cueilleurs des régions où ont été domestiqués pour la première fois le manioc et la téosinte. Ces observations laissent supposer que l'expansion de $\mathrm{Di}^{\mathrm{a}}$ en Amérique Centrale et Sud ait été concomitante à celle des langues et des modes de subsistance pendant la période précolombienne. Certains mécanismes bien documentés d'isolement géographique suivi de dérive génétique importante expliqueraient l'absence de cet allèle dans la région du Chaco (Paraguay), en Terre de Feu et chez les Yanomama. En revanche, son absence commune à l'ensemble des populations panaméennes de langue Chibchan-Paezan et qui n'ont domestiqué le maïs que plus tard par acculturation, rappelle combien ces populations représentent encore une énigme dans le contexte du peuplement premier de l'Amérique Centrale et du Sud. En Amérique du Nord, son absence chez l'ensemble des populations de langue Eskimo-Aleut et Na-Dene est cohérente avec un modèle de peuplement de l'Amérique en plusieurs vagues. En effet, si l'ensemble des populations natives d'Amérique semblent descendre d'une première vague, porteuse de l'antigène $\mathrm{Di}^{\mathrm{a}}$, de nombreux éléments linguistiques et génétiques récents indiquent que les populations actuelles EskimoAleut et Na-Dene descendent du croisement entre cette première vague et des impulsions plus tardives de populations non-porteuses de $\mathrm{Di}^{\mathrm{a}}$, venues de Sibérie et confinées à la frange arctique de l'Amérique du Nord. Par ailleurs, $\mathrm{Di}^{\mathrm{a}}$ est absent des populations de langues Yenisseiennes de Sibérie. Or les linguistes supposent que ces langues sibériennes sont parentes des langues amérindiennes Na-Dene du nord-ouest de l'Amérique du Nord. L'absence de $D i^{a}$ serait alors un élément plaidant en faveur de cette parenté linguistique (Diamond, 2011). 


\section{Quand gènes et cultures mongoles couvrirent l'Asie}

L'étude des mécanismes à l'origine de la répartition des groupes sanguins n'est pas uniquement fructueuse pour l'Amérique. L'Asie centrale a connu elle aussi d'importantes expansions humaines depuis le Paléolithique, qui ont contribué à la répartition actuelle de nombreuses caractéristiques culturelles et biologiques, en Asie et au-delà (Di Cristofaro et al., 2013). Les documents archéologiques et historiques font état d'une intensification des migrations à l'Age des Métaux, vers le $2^{\mathrm{e}}$ millénaire avant notre ère jusqu'au IV ${ }^{\mathrm{e}}$ siècle avant notre ère, impliquant plusieurs grandes populations nomades des steppes atteignant la Mésopotamie, l'Égypte et qui ont pénétré plusieurs fois en Europe Centrale. Lorsqu'ils régnaient sur la plus grande partie des steppes d'Asie centrale, les Scythes de l'Âge du Fer ont joué un rôle important dans l'établissement du commerce transcontinental, notamment la Route de la Soie. Après leur déclin, les cavaliers nomades ont prospéré dans l'Altaï, puis migré vers $l^{\prime} E u-$ rope Centrale où ils se sont mêlés aux populations Franques. Ces expansions s'achèvent avec les Huns qui atteignirent $l^{\prime}$ Europe au $\mathrm{IV}^{\mathrm{e}}$ siècle de notre ère et les khans mongols qui constituèrent les empires les plus étendus (III ${ }^{\text {e }}$ siècle avant notre ère - $\mathrm{XIII}{ }^{\mathrm{e}}$ siècle de notre ère) (Anthony, 2007).

Sur le plan linguistique, les différents nomades des steppes ont été successivement indo-européens, finnoougriens et altaïques (Ruhlen, 1987).
Ces dernières langues comprennent au moins les familles turques, mongoliques et toungouses et sont parlées de la Turquie à l'Extrême-Orient russe. Sur le plan génétique, certaines lignées masculines seraient héritées de ces expansions linguistiques indo-européennes et altaïques, et leur maintien sur plusieurs générations assuré par le nombre plus important de partenaires - et donc de descendants - pour certains hommes de ces sociétés expansives car ils jouissaient d'un plus grand prestige social ${ }^{4}$ (Balaresque et al., 2015). Enfin, à l'instar des populations amérindiennes, les populations centrasiatiques possèdent l'antigène $\mathrm{Di}^{\mathrm{a}}$ et l'aire couverte par ce dernier coïncide aux répartitions des langues Altaïques et aux extensions maximales des territoires conquis par les Mongoles.

La réappréciation de la répartition géographique $\mathrm{du}$ groupe sanguin Diego et certaines explorations de biologie moléculaire montre de grandes variations dans les gammes de fréquences. On trouve notamment, une corrélation positive significative entre les fréquences de Diego et les coordonnées géographiques témoignant également d'une diffusion à partir de la Mongolie et du nord-est de la Chine par des nomades pastoraux locuteurs de l'Altaïque (Petit et al., 2019). En Inde, l'antigène $\mathrm{Di}^{\mathrm{a}}$ n'a été détecté que dans les populations du nord (Bhil Madhya, Rajbanshi Bengali, Bihar, Oraon et Punjab) et plus au nord de l'Inde (Nosherpa, Népal, Sindhi, Balochi et 
Hazara, Pakistan), avec des fréquences nulles dans le sud (Tamil Nadu, Irula et Kurumba) et le sud-est. Cette partition Nord/Sud s'expliquerait par les conquêtes post-expansion mongole. L'Empire Moghol (1526-1857) a été fondé dans le nord de l'Inde en 1526 par Babur, descendant de Tamerlan (1336-1405), premier souverain de la dynastie Timuride, communément considéré comme le conquérant nomade Turco-Mongol de la steppe eurasienne et de Gengis Khan. À la fin du règne d'Akbar en 1605, l'Empire s'étendait du Pendjab, du Bengale au Gujarat. Certains territoires ont

\section{Conclusion}

La façon dont se répartit la diversité génétique et culturelle peut donner des indications sur la façon dont les populations se sont déplacées, se sont rencontrées et se sont adaptées. Ces exemples sur la répartition du groupe sanguin Diego en Amérique et en Asie illustrent comment, à partir d'un unique marqueur biologique identifié il y a un demi-siècle, il est possible d'obtenir des informations suffisantes et déterminantes, qui, une fois comparées aux données historiques et culturelles, permettent de tirer des conclusions fines sur l'histoire des peuplements. Ainsi, cet exemple nous permet de rebondir sur deux points importants. Le premier, plus général, est que pour répondre aujourd'hui à des problématiques d'anthropologie il est indispensable de été conquis vers le sud au milieu du XVII ${ }^{e}$ siècle mais les données génétiques actuelles n'ont pas permis d'en détecter l'héritage biologique. Enfin, dans le monde, très peu d'individus sans ascendance asiatique possèdent l'antigène $\mathrm{Di}^{\mathrm{a}}$. Sa présence sporadique dans les régions limitrophes de l'expansion mongole, comme en Pologne qui a été envahie par les Tartares mongols au XIII ${ }^{\mathrm{e}}$ siècle et entre le $\mathrm{XV}^{\mathrm{e}}$ et le XVII ${ }^{\mathrm{e}}$ siècle et en Afghanistan et au Pakistan à proximité des Hazara ${ }^{5}$, dont l'ethnogenèse les place comme les descendants des Mongoles, pourrait provenir d'un métissage.

passer par une approche interdisciplinaire. Le deuxième, plus spécifique, est que parmi la palette de marqueurs moléculaires qui sont à la disposition des généticiens, il n'est pas toujours utile d'étudier des marqueurs génétiques dits " modernes » (car issus de nouvelles générations de séquençage haut débit) pour répondre à des problématiques bio-culturelles. Comme dans toute discipline, comme dans tout métier, il faut trouver le bon outil. Pour paraphraser cela, nous pourrions dire qu'il n'est pas utile d'utiliser la bombe atomique pour tuer une mouche si la tapette suffit...

Outre ces aspects de peuplement, la répartition différentielle des groupes sanguins à l'échelle planétaire pose des problèmes de santé publique. Dans un contexte actuel de mondialisation 
induisant la rencontre de populations à l'origine géographiquement, biologiquement et culturellement éloignées, des incompatibilités de transfusions sont déclinées. Il est donc indispensable de connaître les zones de polymorphismes immunogènes pour assurer la sécurité transfusionnelle. La description et la compréhension de la distribution géographique actuelle des systèmes de groupes sanguins nécessitent, comme nous l'avons $\mathrm{vu}$, une approche pluridisciplinaire et sont de ce fait des problématiques clefs de l'Anthropologie bioculturelle.

\section{Bibliographie}

Anthony DW. 2007,The Horse, the Wheel, and Language: How Bronze-Age Riders from the Eurasian Steppes Shaped the Modern World, Princeton, Princeton University Press.

Bégat C., Bailly P., Chiaroni J., Mazières S. 2015, « Revisiting the Diego Blood Group System in Amerindians: Evidence for Gene-Culture Comigration ", dans PLoS One, nº 6, vol. X t. 7 : e0132211.

Bailly P., Chiaroni J., Roubinet F. 2015, Les groupes sanguins érythrocytaires, Montrouge, John Libbey Eurotext éditions.

Balaresque P., Poulet N., Cussat-Blanc S., Gerard P., Quintana-Murci L., Heyer E., Jobling M.A. 2015, « Y-chromosome descent clusters and male differential reproductive success: young lineage expansions dominate Asian pastoral nomadic populations ", dans Eur J Hum Genet, n 23, vol. $X: 1413-1422$.

Bernard J. 1983, Le Sang et l'Histoire, Paris, Buchet Chastel.
Chiaroni J., Chevé D., Berland-Benhaim C., Le Coz P. 2016, Le Sang : donner et recevoir, Paris, CNRS Editions..

Diamond J. 2011, " Deep relationships between languages ", dans Nature, $\mathrm{n}^{\circ} 476$ : 291-292.

Di Cristofaro J., Pennarun E., Mazières S., Myres N.M., Lin A.A., Temori S.A., Metspalu M., Metspalu E., Witzel M., King R.J., Underhill P.A., Villems R., Chiaroni J. 2013, « Afghan Hindu Kush: Where Eurasian sub-continent gene flows converge", dans PLoS ONE, nº 8, t. X : e76748.

Greenberg JH., Turner CG., \& Zegura SL. 1986, " The settlement of the Americas: a comparison of the linguistic, dental, and genetic evidence ", dans Current Anthropo$\log y, \mathrm{n}^{\circ} 27$ : 477-497.

Junqueira PC., Castilho L. 2002, " The history of the Diego blood group ", dans Rev. Bras. Hematol. Hemoter., n 24, vol. I : 15-23.

Mazières S. 2011, " Towards a reconciling model about the initial peopling of America ", dans C R Biol., n 334, vol. VII : 497504.

Petit F., Minnai F., Chiaroni J., Underhill P.A., Bailly P., Mazières S., Costedoat C. 2019, «The radial expansion of the Diego blood group system polymorphisms in Asia: Mark of co-migration with the Mongol conquests ", dans Eur J Hum Genet, n²7, vol. I : 125-132.

Reich D., Patterson N., Campbell D., Tandon A., Mazieres S., Ray N., Parra MV., Rojas W., Duque C., Mesa N., García L.F., Triana O., Blair S., Maestre A., Dib J.C., Bravi C.M., Bailliet G., Corach D., Hünemeier T., Bortolini M.C., Salzano F.M., Petzl-Erler M.L., Acuña-Alonzo V., Aguilar-Salinas C., Canizales-Quinteros 
S., Tusié-Luna T., Riba L., RodríguezCruz M., Lopez-Alarcón M., Coral-Vazquez R., Canto-Cetina T., Silva-Zolezzi I., Fernandez-Lopez J.C., Contreras A.V., Jimenez-Sanchez G., Gómez-Vázquez M.J., Molina J., Carracedo A., Salas A., Gallo C., Poletti G., Witonsky D.B., Alkorta-Aranburu G., Sukernik R.I., Osipova L., Fedorova S.A., Vasquez R., Villena M., Moreau C., Barrantes R., Pauls D., Excoffier L.,
Bedoya G., Rothhammer F., Dugoujon JM., Larrouy G., Klitz W., Labuda D., Kidd J., Kidd K., Di Rienzo A., Freimer N.B., Price A.L., Ruiz-Linares A. 2012, "Reconstructing Native American population history", dans Nature, $\mathrm{n}^{\circ}$ 16, vol. CDLXXXVIII, $\mathrm{t}$. 7411 : 370-374.

Ruhlen M. 1987, A Guide to the World's Languages: Volume I, Classification, Stanford, Stanford University Press.

Caroline COSTEDOAT est Maître de conférences-HDR en génétique des populations à Aix- Marseille Université au sein de l'UMR ADES 7268 (AMU, CNRS, Établissement Français du Sang PACA Corse). Ces activités de recherches en anthropologie génétique se concentrent notamment sur l'étude des relations génome-environnement et sur des contextes d'identification populationnelle et individuelle du passé.

caroline.costedoat@univ-amu.fr

Stéphane MAZIERES est anthropologue généticien, Chargé de recherche au CNRS au laboratoire ADES, UMR7268 (Aix Marseille Université, CNRS, Etablissement Français du Sang PACA Corse). Ses travaux portent essentiellement sur l'étude de la diversité génétique humaine, la dynamique des peuplements, et l'étude de la répartition des polymorphismes du globule rouge entre les

populations.

stephane.mazieres@univ-amu.fr

\section{Notes}

1 Charles Salmon (1925-2009), médecin, immunologiste et hématologiste estimait que sur la seule base des groupes sanguins, chaque individu constitue une solitude biologique à tel point qu'il existe plus de combinaisons de phénotypes sanguins que d'êtres humains s'étant succédé sur Terre depuis l'origine de l'Homme anatomiquement moderne.

2 En 2013, on dénombrait 30 à 35 études majeures dans l'année, soit une fréquence d'une étude tous les 11 jours !

3 Une caractéristique remarquable à l'échelle mondiale est que tous les Amérindiens d'Amérique Centrale et du Sud ont un phénotype $\mathrm{ABO}-\mathrm{RH}$ unique, $\mathrm{O}+$. Ceci résulterait d'un effet fondateur important lors du peuplement initial, accentué en Amérique du Sud par la percolation au goutte-à-goutte de l'Isthme de Panama. Cependant, il a été montré en Bolivie une prévalence des parasites intestinaux et que ces infestations pouvaient induire une surproduction d'anticorps anti-A, -B particulièrement puissants, chez l'hôte. L'élimination de gamètes autres que $\mathrm{O}$ par ces anticorps n'est donc pas à exclure. Or à ce jour ; l'hypothèse de sélection du phénotype $\mathrm{O}+$ chez les Amérindiens demeure vierge de travaux depuis 1967.

4 La lignée génétique masculine associée aux Mongoles a connu une telle expansion démographique en Eurasie que 16 millions d'hommes, soit près de $0,5 \%$ de la population mondiale, possèdent le même chromosome $Y$.

5 « Hazara " proviendrait du persan « hezār » signifiant « mille », puisque les Mongoles laisseraient un millier de soldats pour assoir leur autorité. Notez par ailleurs que si les Mongoles parlent l'altaïque, les Hazaras parlent aujourd'hui une langue indo-européenne. 